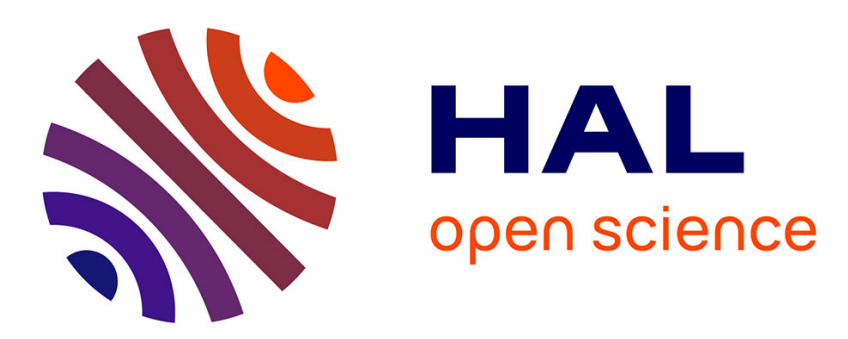

\title{
IMPORTANCE OF UPPER-LIMB INERTIA IN CALCULATING CONCENTRIC BENCH PRESS FORCE
}

Olivier Rambaud, Abderrahmane Rahmani, Bernard Moyen, Muriel Bourdin

\section{- To cite this version:}

Olivier Rambaud, Abderrahmane Rahmani, Bernard Moyen, Muriel Bourdin. IMPORTANCE OF UPPER-LIMB INERTIA IN CALCULATING CONCENTRIC BENCH PRESS FORCE. Aristotelian Society Supplementary Volume, 2008, 22. hal-02510452

\section{HAL Id: hal-02510452 \\ https://hal.science/hal-02510452}

Submitted on 17 Mar 2020

HAL is a multi-disciplinary open access archive for the deposit and dissemination of scientific research documents, whether they are published or not. The documents may come from teaching and research institutions in France or abroad, or from public or private research centers.
L'archive ouverte pluridisciplinaire HAL, est destinée au dépôt et à la diffusion de documents scientifiques de niveau recherche, publiés ou non, émanant des établissements d'enseignement et de recherche français ou étrangers, des laboratoires publics ou privés. 


\title{
ImPortance OF UPPER-Limb IneRTia in CALCUlating Concentric Bench Press Force
}

\author{
Olivier Rambaud, ${ }^{1}$ Abderrahmane Rahmani, ${ }^{2}$ Bernard Moyen, ${ }^{1,3}$ and Muriel Bourdin ${ }^{1}$ \\ ${ }^{1}$ Biomechanics and Impact Mechanics Laboratory, Claude Bernard University Lyon 1, Lyon-South Teaching Hospital, Oullins; \\ ${ }^{2}$ Laboratory of Physical and Sports Activities, University of the Maine, Le Mans; ${ }^{3}$ Department of Orthopedic and Traumatology \\ Surgery and Sports Medicine, Lyon-South Teaching Hospital, Pierre Benite, France
}

\begin{abstract}
The purpose of this study was to investigate the influence of upperlimb inertia on the force-velocity relationship and max- imal power during concentric bench press exercise. Reference peak force values $\left(F_{\text {peak }} p\right)$ measured with a force plate positioned below the bench were compared to those measured simultaneously with a kinematic device fixed on the barbell by taking $\left(F_{\text {peakt }}\right)$ or not taking ( $F_{\text {peakb) }}$ upper-limb inertia into account. Thirteen men (27.8 64.1 years, $184.665 .5 \mathrm{~cm}, 99.5618 .6 \mathrm{~kg}$ ) performed all-out concentric bench press exercise against 8 loads ranging between 7 and 74 $\mathrm{kg}$. The results showed that for each load, $F_{\text {peak }}$ was significantly less than $\mathrm{F}_{\text {peak }}(P, 0.0001)$, whereas no significant difference was

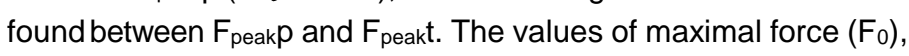
maximal velocity $\left(\mathrm{V}_{0}\right)$, optimal velocity $\left(\mathrm{V}_{\mathrm{opt}}\right)$, and maximal power $\left(P_{\text {max }}\right)$, extrapolated from the force-and power-velocity relationships determined with the kinematic device, were significantly underestimated when upper-limb inertia was ignored. The results underline the importance of taking account of the total inertia of the moving system to ensure precise evaluation of upper-limb muscular characteristics in all-out concentric bench press exercise with a kinematic device. A major application of this study would be to develop precise upper-limb muscular characteristic evaluation in laboratory and field conditions by using a simple and cheap kinematic device.
\end{abstract}

\section{KEYWORDS}

force-velocity relationship, maximal power, ballistic movement

\section{INTRODUCTION}

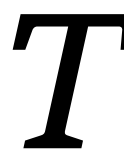

he force-velocity relationship and explosive maximal power output $\left(P_{\max }\right)$ are widely used parameters in studying the mechanical characteristics of muscles or muscle groups during cycling $(1,26,32)$ and single- $(22,30)$ or multiple-joint lower-limb extension (25). In explosive events, neuromuscular and mechanical factors, including maximal lower-limb force and power, may contribute to final performance (31). Several authors have described significant relationships between $P_{\max }$ and explosive event performance in track cycling (12) and sprint running (17). Maximal lower-limb strength has been related to sprint running performance $(4,40)$. Hautier et al. (13) reported that optimal velocity $\left(\mathrm{V}_{\mathrm{opt}}\right)$ (i.e., the velocity corresponding to $P_{\max }$ ) correlates to the percentage of fast twitch muscle fibers in the vastus lateralis, and $V_{\text {opt }}$ has been related to track cycling performance (12).

A prime objective in training is to identify the best training program and conditions to enhance the athletes' performance (36). Athletes usually train at a certain percentage of the maximum weight they can lift once with proper form and technique (1RM). Load, however, is not the only parameter taken into account in strength training programs, especially for explosive events. Several authors have shown that training with heavy loads enhances strength, whereas training with light loads enhances power production $(5,15,16)$. Both qualities are important in performance. It has previously been shown that maximal power is obtained for light loads (i.e., high movement velocity) $(2,25,28)$. A precise deter- mination of the load associated with $P_{\max }$ could be useful for improving training efficiency. This suggests that muscular characteristic evaluation for athletes involved in explosive events should concern maximal force and maximal power production.

Maximal power can be determined by using kinematic systems, which enable muscular parameters to be assessed in terms of lifted load displacement during squat $(14,25)$ or bench press exercise $(6-11,14,27)$. To calculate force from kinematic data accurately, the total inertia of the mechanical system must be determined accurately (i.e., the mass of the lifted load plus the inertia of the levers and body segments). Several authors have shown that the force produced during single-joint extension of the lower limbs is underestimated if lever arm and leg inertia are not taken into account $(20,23,39)$, and an underestimation of $P_{\max }$, maximal force, and maximal velocity extrapolated from the force-velocity and power-velocity relationships can result (23).

In various bench press studies $(6-11,14,27)$, force was calculated on the basis of load only, without taking into account the total inertia of the system (i.e., load plus upper- limb mass). This means that the upper-limb mass and the effort required to accelerate the upper limbs were neglected, and this disregard led to an underestimation of maximal power production. This VOLUME 22 | NUMBER 2 | MARCH 2008 | 383 
methodological bias would explain why mean maximal force values obtained in groups of team- sport players using kinematic devices $(11,14)$ were system- atically less than those measured with a force plate $(18,34,35,37)$.

It would be of interest for throwers or team-sport players to assess upper-limb muscular characteristics. The purpose of this study was twofold. First, it sought to determine the in- fluence of upper-limb inertia on bench press force calculation by comparing peak force values obtained directly with a force plate to those calculated from barbell displacement recorded with a kinematic system taking upper-limb inertia into account and not taking it into account. Second, the influence of inertia on the force-velocity and power-velocity relation- ships was examined to propose a valid protocol to evaluate upper-limb muscular characteristics accurately.

\section{METHODS}

Experimental Approach to the Problem

Subjects came to the laboratory once. During the session, a series of bench press movements was performed against 8 loads $(7,15,24,34,44,54,64$, and $74 \mathrm{~kg}$ ), with simultaneous force plate and kinematic recoding (Figure 1). The force- velocity and powervelocity relationships were determined from the kinematic data. Correlations between results under various measurement conditions were analyzed statistically.

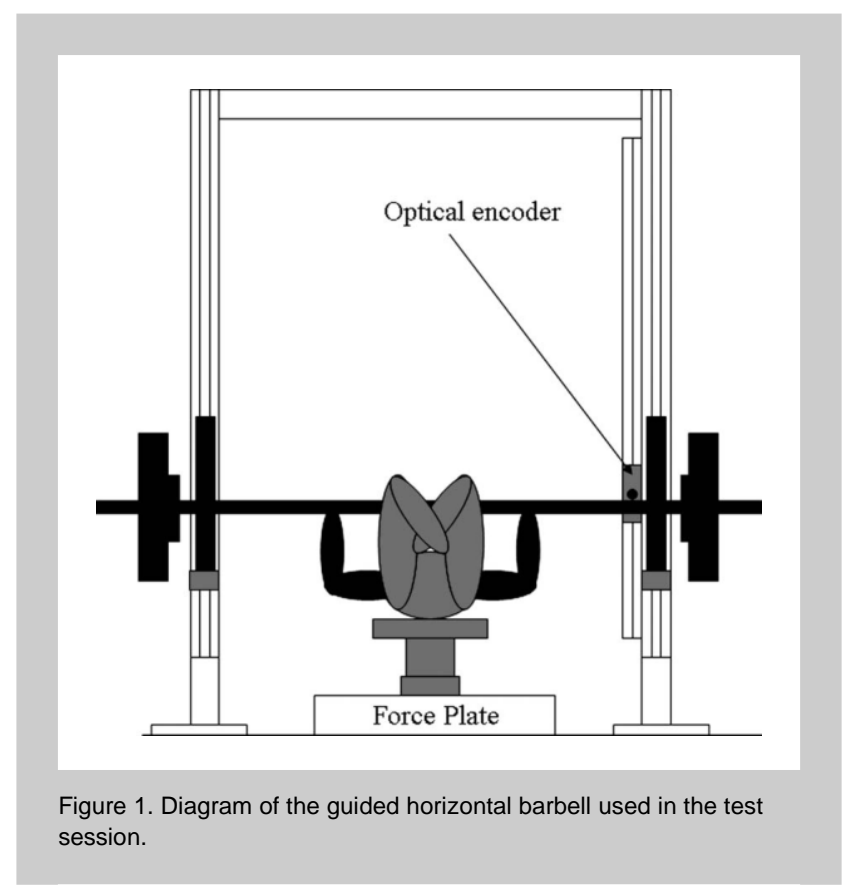

\section{Subjects}

Thirteen men (mean age, 27.864 .1 years; mean height, $184.665 .5 \mathrm{~cm}$; mean body mass, 99.5618 .6 $\mathrm{kg}$ ) were enrolled. They were all interregional to national level discus $(n=8)$ and hammer $(n=5)$ throwers, who had been doing regular strength training and, in particular, bench press exercise for at least 3 years. The study took place at the beginning of the competition 384 season, so that subjects would be homogenous in terms of training status. The subjects were instructed to avoid strength training the day before laboratory testing and to cancel training on the day itself. In accordance with Lyon Ethics Committee guidelines, the study protocol was explained to the subjects before they gave their written informed consent.

\section{Procedures}

Upper-limb force was assessed on a series of bench press movements made with a guided horizontal barbell against increasing loads $(7,15,24,34,44,54,64$, and $74 \mathrm{~kg}$ ). The barbell, the mass of which totaled $24 \mathrm{~kg}$ including the guidance system, was modified to obtain the lightest ( 7 and $15 \mathrm{~kg}$ ) loads; both ends of the barbell were taken off for the $15-\mathrm{kg}$ load, and the barbell as such was replaced by an aluminum tube of the same diameter for the 7-kg load. This protocol allowed each subject to be compared for a given load.

The test session began with a general warm-up involving several sets of bench press exercise at low loads. Subjects lay supine on the bench (Figure 1). The barbell was positioned across their chest at nipple level above the pectoralis major, supported by the lower mechanical stops of the measure- ment device. At the start of the movement, the shoulders had to stay in contact with the bench. The subjects held the barbell at shoulder width, with an initial elbow angle of $90^{\circ}$ between the upper arm and forearm $(14,29)$, as checked with a protractor, and the barbell was marked so as to be held at exactly the same place in all trials. The subjects held their legs crossed above the bench. On a spoken order, the subject applied force as fast as possible to perform an explosive concentric arm extension. The subjects did not have to lower the bar to their chest, just to explode it off the chest as rapidly as possible. The barbell had to stay in the subjects' hands throughout the movement and could not be thrown. Two trials were performed at each load. Each trial was followed by a rest period of at least 3 minutes. The best trial, in terms of force peak as measured by the force plate, was used for analysis.

\section{Measuring System}

All lifts were performed by using the same guided horizontal barbell (Multipower Basic; Panatta Sport, Apiro, Italy) and allowing only vertical movement. Extra load was added by adding weight plates equally to both ends of the bar.

Variation in the vertical force during the movement was recorded by a Kistler force plate (Kistler type 9281 Kistler Instrumente AG, Winterthur, Switzerland). Analog signals from the force plate were amplified by charge amplifiers (Kistler type 9861 A; Kistler Instrumente AG). The force plate was mounted according to the manufacturer's specifications and had been calibrated by the manufacturer; no periodic recalibrating was necessary. The force plate was fixed to the ground with a base provided by the manufacturer. The bench was also fixed onto the force plate. The force signal was linear $(<0.5 \%)$ over a range of force of 0 to $10 \mathrm{kN}$, to within $\pm 1 \%$. The resonant frequency of the force platform was greater than $200 \mathrm{~Hz}$. The amplifiers were reset to 0 after the subject took his place on the bench. $F_{\text {peak }} p$ designates the peak force values recorded by the force plate during the movement. The displacement signal was recorded by a valid and reliable kinematic system $(3,24)$, consisting of 2 infrared photo interrupters, locked in a shuttle that glided on a track bar (Figure 1) and was fixed onto the barbell. The optical encoder was placed facing an optical code strip 
that was stuck to the track bar and composed of slots $0.75 \mathrm{~mm}$ apart. The optical encoder counted the slots as it passed them and recorded each $0.75-\mathrm{mm}$ displacement as the load was being raised by the subject. Displacement was recorded over a maximum distance of $2,000 \mathrm{~mm}$ with a minimum speed of 0.008 $\mathrm{m} \cdot \mathrm{s}^{-1}$. The displacement signal was stored on a personal computer (486 DX2, $66 \mathrm{MHz}$ ). Customized software calculated the instantaneous velocity and acceleration from successive displacement time-derivatives for each lift. In- stantaneous force ( $F$, measured in newtons) was calculated as follows: $F=M(a+g)+F_{f}$; where $M$ is the moving mass; $g$ is the gravitational acceleration $\left(9.81 \mathrm{~m} \cdot \mathrm{s}^{-}\right.$ $\left.{ }^{2}\right)$; $a$ is the calculated acceleration $\left(\mathrm{m} \cdot \mathrm{s}^{-2}\right)$; and $\mathrm{F}_{f}$ is the friction force determined by a freefall test added to the concentric phase. $\mathrm{F}$ was determined by taking into account only the barbell load $\left(F_{\text {peak }} b\right)$ or the combined mass of the load plus the upper limbs $\left(F_{\text {peakt }}\right)$, as estimated from Winter's anthropometric tables (38). The index $b$ is used here and throughout to signify that upper-limb mass was not taken into account, and the index $t$ is used to indicate that it was. The instantaneous power, measured in watts, was calculated as the product of force and velocity at any given time. The calculation is detailed in work published by Bosco et al. (3).

The displacement and force signals were sampled simul- taneously $(200 \mathrm{~Hz})$ and stored on a personal computer (486 DX2, $66 \mathrm{MHz}$ ) by an electronic interface card equipped with a 12-bit counter (type HCTL-2000; Hewlett Packard, Palo Alto, CA) for the kinematic device and a 12-bit A/D converter card (type PC-LPM16; National Instrument France, Le Blanc-Mesnil, France) for the force plate. The signals were digitally filtered with a 12-Hz low-pass Butterworth filter with no phase lag.

Force-Velocity and Power-Velocity Relationships The relationships between force and velocity and between power and velocity were determined from the kinematic data for each subject. For each load, the peak force and corresponding velocities were recorded (23).
The force- velocity relationships were described by linear regression between the force and the velocity of the movement for each load lift; a nonlinear forcevelocity model would not have increased correlation coefficient values consistently. The theoretical maximal isometric force $\left(F_{0}\right)$ and maximal contraction velocity $\left(\mathrm{V}_{0}\right)$, corresponding to the force-velocity curve intercepts with the force and velocity axes, respectively, were extrapolated from the curve and annotated as $\mathrm{F}_{0} \mathrm{~b}$ or $\mathrm{F}_{0} \mathrm{t}$ for force and $V_{0} b$ or $V_{0} t$ for velocity, depending on the mass definition used. For each load, the peak power values and corresponding velocity were recorded (23). The power- velocity relationships were described by a second-order polynomial regression. $\mathrm{P}_{\max }$ and $\mathrm{V}_{\text {opt }}$ were extrapolated from the curve and annotated as $P_{\max } b$ or $\mathrm{P}_{\text {maxt }}$ for power and $\mathrm{V}_{\mathrm{opt}} \mathrm{b}$ or $\mathrm{V}_{\mathrm{opt}} \mathrm{t}$ for velocity, depending on the mass definition used.

\section{Statistical Analyses}

Data are presented as mean $\pm S D$. The statistical significance threshold was set at $P \leq 0.05$. In agreement with statistical norms (19), trial-to-trial reliability was tested on $F_{\text {peak }} p$ by assessing the intraclass correlation coefficient (ICC) between the 2 trials recorded with the force plate. The ICC and statistical power were calculated from analysis of variance with repeated measures.

The Pearson product-moment correlation coefficient $(r)$ was used to compare $F_{\text {peakt }}$ and $F_{\text {peak }} p$ and to determine the force-velocity and power-velocity relationships. The Wil- coxon test was used to compare force values per load under the various measurement and calculation conditions and to compare parameters determined on the basis of the force- velocity and power-velocity relationships, including upper- limb inertia or not.

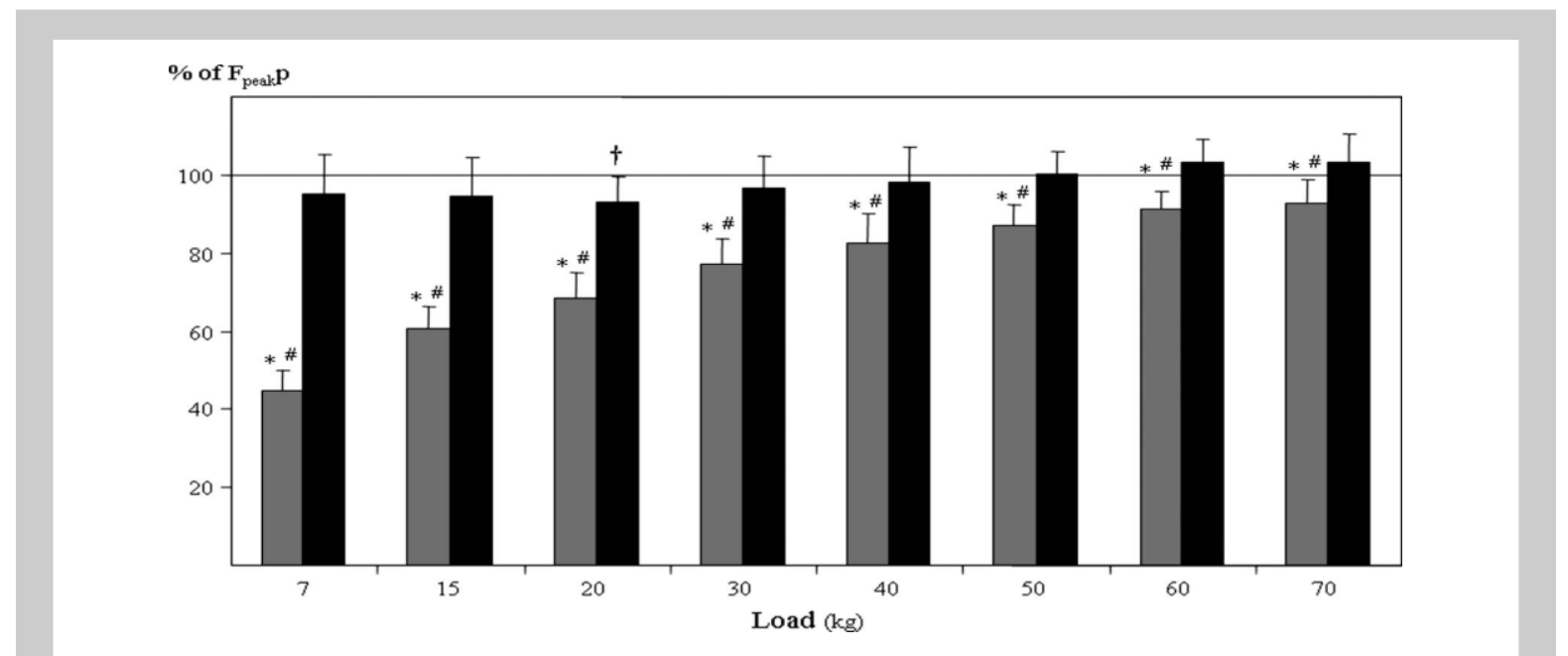

Figure 2. Force peak values taking and not taking upper-limb inertia into account ( $F_{\text {peakt }}$ in black and $F_{\text {peak }} b$ in gray, respectively) related to reference force peak values $\left(F_{\text {peak }} P\right)$ for each experimental load. * Significanty different from $100 \% F_{\text {peak }}(P<0.0001)$. $\dagger$ Significantly different from $100 \%$ $F_{\text {peak }}(P<0.01)$. \# $F_{\text {peak }} b$ significantly different from $F_{\text {peakt }}(P<0.0001)$ 


\section{RESULTS}

Reliability

The trial-to-trial ICC for $F_{\text {peak }}$ was 0.92 , and the statistical power was $97.8 \%$. According to statistical norms (33), the reliability coefficient was very good, with no statistical difference between the 2 trials. This good intertrial reproducibility shows that subjects were accustomed to the bench press and justifies the choice of the best peak-force trial for analysis.

Importance of Inertia

The mean upper-limb mass determined according to Winter (38) was $8.7 \pm 1.6 \mathrm{~kg}$. The values of $F_{\text {peak }} b$ and $F_{\text {peakt }}$ were expressed as a percentage of $F_{\text {peak }} p$ (Figure 2). $F_{\text {peak }} p$ and $F_{\text {peakt }}$ were significantly greater than $\mathrm{F}_{\text {peak }} \mathrm{b}$, regardless of the load $(P<0.0001)$. The difference between $F_{\text {peak }}^{\%}$ and $F_{\text {peak }} b$, expressed as a percentage of $F_{\text {peak }} p$, decreased with increasing load, from $55.72 \pm 5.41 \%$ to $8.44 \pm 2.10 \%$ for $7 \mathrm{~kg}$ and $74 \mathrm{~kg}$ respectively. $F_{\text {peakt }}$ and $F_{\text {peak }} p$ did not significantly differ, except at $24 \mathrm{~kg}(P<0.01)$. F peakt correlated significantly with $\mathrm{F}_{\text {peakp }}(r=0.91 ; P<0.0001)$ (Figure 3).

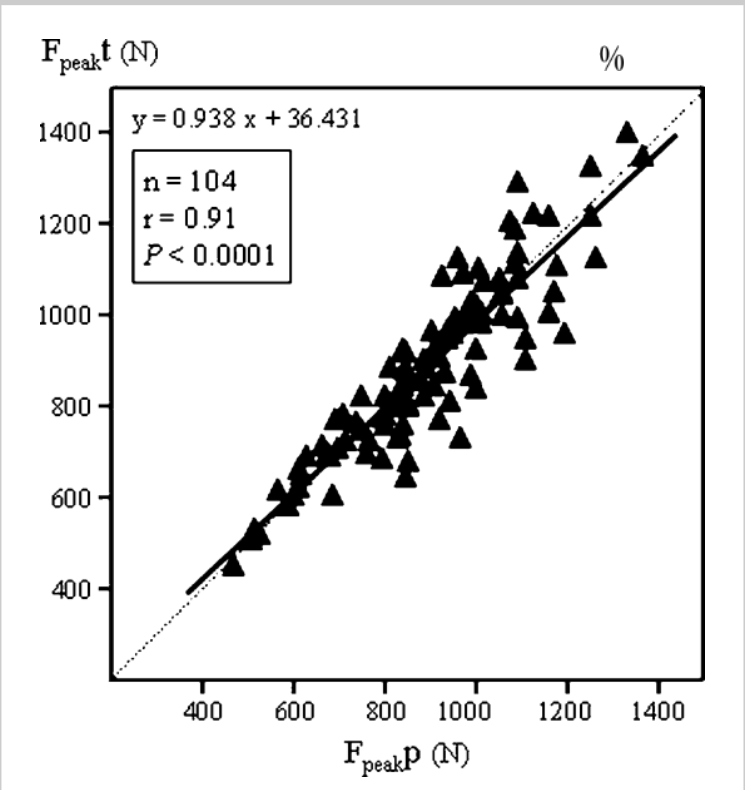

Figure 3. Relationship between force peak values measured with the force plate $\left(F_{\text {peak }} p\right)$ and peak force values calculated from data obtained with the kinematic device taking upper limb inertia into account $\left(F_{\text {peak }} t\right)$. The dotted line represents the identity line.

Force-Velocity and Power-Velocity Relationships The mean force-velocity and power-velocity relationships are shown in Figure 4. The force-velocity relationships were significantly linear (Figure 4a), regardless of whether upper- limb mass was included in the calculation $(0.75<\mathbf{r}<0.98 ; P<0.05)$ or not $(0.77$ $<\boldsymbol{r}<0.99 ; P<0.05)$. Vob was significantly less than Vot $\left(2.11 \pm 0.41 \mathrm{~m} \cdot \mathrm{s}^{-1}\right.$ and $3.67 \pm 1.06 \mathrm{~m} \cdot \mathrm{s}^{-1}$, respectively; $P<0.01$ ), and $F_{0} b$ was significantly less than $F_{0} t(1,037.4 \pm 206.6 \mathrm{~N}$ and $1,100.2 \pm 200.1 \mathrm{~N}$, respectively; $P<0.01$ ).

The power-velocity relationships significantly matched a second-order function (Figure 4b), regardless of whether upper-limb mass was included in the calculation $(0.88<\boldsymbol{r}<0.99 ; P<0.05)$ or not $(0.96<\boldsymbol{r}<0.99$; $P<0.05)$. $P_{\max }$ b was significantly less than $P_{\max } t(1,077.8$ $\pm 291.2 \mathrm{~W}$ and $1,598.9 \pm 435.7 \mathrm{~W}$, respectively; $P<0.01)$. Likewise, $\mathrm{V}_{\text {optb }}$ was significantly less than $\mathrm{V}_{\text {opt }}(1.95 \pm$ $0.21 \mathrm{~m} \cdot \mathrm{s}^{-1}$ and $3.04 \pm 0.53 \mathrm{~m} \cdot \mathrm{s}^{-1}$, respectively; $P<0.01$ ).

\section{DISCUSSION}

This study shows the importance of taking upper-limb inertia into account if force and power production during all-out bench press exercise is to be evaluated accurately with a kinematic device. When upper-limb mass was ignored, the force calculated with the kinematic device, regardless of the load, was significantly less than that measured with the force plate (Figure 2). This underestimation was greater for lighter loads because the relative contribution of the upper-limb mass to the total inertia decreased as the lifted load increased, from $53.73 \pm 4.78 \%$ to $10.38 \pm 1.74 \%$ at $7 \mathrm{~kg}$ and $74 \mathrm{~kg}$ respectively. $V_{0}, F_{0}, P_{\max }$, and $V_{o p t}$ were significantly under- estimated, by $40.9 \%, 5.9 \%, 32.1 \%$, and $34.9 \%$ respectively, when upper-limb inertia was not taken into account.

The percentage error was particularly great in the case of maximal power and velocity parameters values (i.e., $\mathrm{V}_{0}$ and $\mathrm{V}_{\mathrm{opt}}$ ) because maximal power is obtained for light loads (i.e., high velocities). For the lightest loads, calculated peak force values were underestimated by $56 \%$ and $41 \%$ for 7 and $15 \mathrm{~kg}$, respectively (Figure 2), when total inertia was not taken into account. These findings support those of Rahmani et al. (23) and Winter et al. (39), who found percentage errors on force values varying from $6 \%$ to $45 \%$, depending on the mass and velocity conditions, when total inertia was not taken into account. Failure to consider upper-limb inertia makes it difficult to compare maximal power and optimal velocity between subjects or populations differing in upper-limb mass. Moreover, the load corresponding to maximal power production varied, from a mean of $36 \mathrm{~kg}$ when upper-limb inertia was ignored to a mean $15 \mathrm{~kg}$ when it was taken into account. This finding has practical consequences in the context of training, particularly for training programs using $P_{\max }$ loading to enhance maximal power. The results also showed that the error on $F_{0}$, though significant, was weak. It may be concluded that taking or not taking upper-limb inertia into account during all-out bench press exercise is of particular importance for determining maximal power and velocity, but of minor interest for determining maximal force.

When upper-limb mass was taken into account, there was no significant difference in force values as measured with the kinematic device or the force plate, except in the case of a 24-kg load, and no significant difference between kinematic and force plate results emerged when all loads were analyzed together without distinction. Moreover, $F_{\text {peak }} P$ and $F_{\text {peakt }}$ were highly correlated, with a regression slope nearly coinciding with the identity line (Figure 3). Fpeakt tended to be less than $F_{\text {peak }} p$ for loads less than $54 \mathrm{~kg}$ but greater for greater loads (Figure 2). 

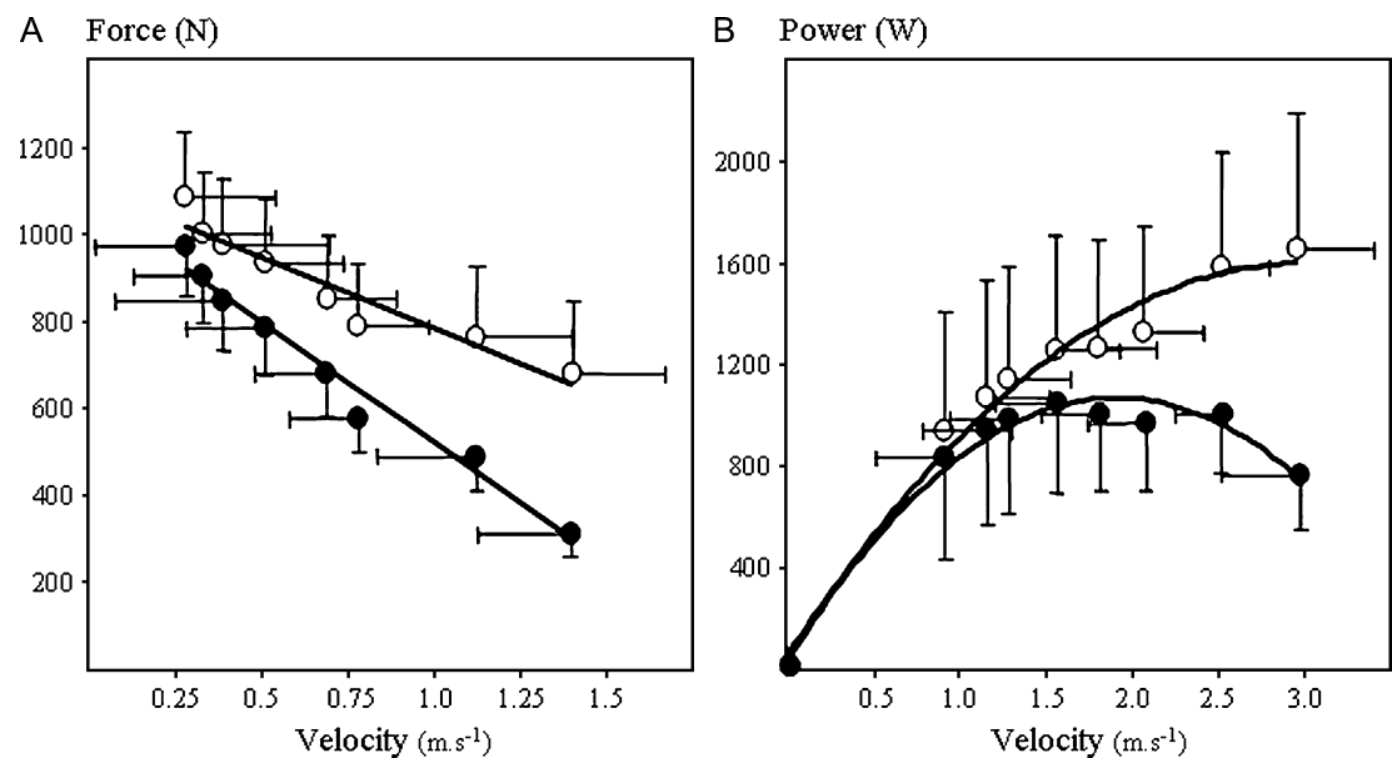

Figure 4. Mean force-velocity (A) and power-velocity (B) relationships taking (open symbols) or not taking (filled symbols) upper-limb mass into account.

This finding probably indicates that the upper-limb mass estimated from the anthropometric tables did not reflect the true inertia. The bench press exercise is a multiplejoint movement involving 2 joints (i.e., the elbow and shoulder); the muscular groups involved come into play progressively, so that inertia may vary during the movement. Complementary studies using a multiplesegmental system to model the upper limbs, including upper- limb inertia, will be necessary to confirm this hypothesis.

Figure 4a,b shows how the shapes of the force-velocity and power-velocity curves are affected when upperlimb inertia is taken into account. These shapes were close to those obtained by Rahmani et al. (25) and Taylor et al. (28). In those studies, maximal power could not be extrapolated accurately from the polynomial regression equation because $P_{\max }$ lay outside the experimental points, in contrast to the current study. Reducing the mass of the original barbell to 7 and $15 \mathrm{~kg}$ enabled a velocity great enough to allow the apex of the power-velocity relationship to be achieved and $P_{\max }$ to be obtained, in contrast to the design by Rahmani et al. $(23,25)$. This raises the main methodological problem encountered in determining maximal power during squat or bench press exercises using a guided barbell. It could be hypothesized that bench throw exercises are more suited to evaluate upper-limb muscular characteristics than bench press exercises are. Newton et al. (21) showed that peak concentric velocities were significantly greater, by $27 \%$, during bench throw than during concentric bench press. Further studies are needed to test this hypothesis, although devices enabling bench throw over a wide load range under safe conditions for the athletes are scarce.

\section{PRACTICAL APPLICATIONS}

This study confirmed the importance of taking the total inertia of the moving system into account to analyze force- velocity and power-velocity relationships accurately during bench press by using a kinematic device. It further highlights the importance of using light loads to enhance the range of velocities in assessing upper-limb muscular parameters to determine $P_{\max }$ accurately. $P_{\max }$ often correlates with per-formance in explosive events; precise determination of $P_{\max }$ would allow interindividual performance variations between throwers to be analyzed. It has a practical application in training; ignoring inertia prevents accurate determination of the load at which an athlete or a practitioner should work to improve his or her power. This protocol allows muscular characteristics to be determined with precision in laboratory and field conditions by using a simple and relatively inex-pensive kinematic device. 


\section{REFERENCES}

1. Arsac, LM, Belli, A, and Lacour, JR. Muscle function during brief maximal exercise: accurate measurements on a friction-loaded cycle ergometer. Eur J Appl Physiol 74: 100-106, 1996.

2. Baker, D. Acute effect of alternating heavy and light resistances on power output during upper-body complex power training. J Strength Cond Res 17: 493-497, 2003.

3. Bosco, C, Belli, A, Astrua, M, Tihanyi, J, Pozzo, R, Kellis, S, Tsarpela, O, Foti, C, Manno, R, and Tranquilli, C. A dynamometer for evaluation of dynamic muscle work. Eur $J$ Appl Physiol 70: 379-386, 1995.

4. Bret, C, Rahmani, A, Dufour, AB, Messonnier, L, and Lacour, $J R$. Leg strength and stiffness as ability factors in $100 \mathrm{~m}$ sprint running. J Sports Med Phys Fitness 42: 274-281, 2002.

5. Caiozzo, VJ, Perrine, JJ, and Edgerton, VR. Traininginduced alterations of the in vivo force-velocity relationship of human muscle. J Appl Physiol 51: 750-754, 1981.

6. Cronin, JB and Henderson, ME. Maximal strength and power assessment in novice weight trainers. J Strength Cond Res 18: 48-52, 2004.

7. Cronin, JB, McNair, PJ, and Marshall, RN. The role of maximal strength and load on initial power production. Med Sci Sports Exerc 32: 1763-1769, 2000.

8. Cronin, JB, McNair, PJ, and Marshall, RN. Magnitude and decay of stretch-induced enhancement of power output. Eur $J$ Appl Physiol 84: 575-581, 2001.

9. Cronin, JB, McNair, PJ, and Marshall, RN. Is velocity-specific strength training important in improving functional performance? J Sports Med Phys Fitness 42: 267-273, 2002.

10. Cronin, JB, McNair, PJ, and Marshall, RN. Power absorption and production during slow, large-amplitude stretch-shorten cycle motions. Eur J Appl Physiol 87: 5965, 2002.

11. Cronin, JB, McNair, PJ, and Marshall, RN. Force-velocity analysis of strength-training techniques and load: implications for training strategy and research. $J$ Strength Cond Res 17: 148-155, 2003.

12. Dorel, S, Hautier, CA, Rambaud, O, Rouffet, D, van Praagh, E, Lacour, JR, and Bourdin, M. Torque and power-velocity relation- ships in cycling: relevance to track sprint performance in world-class cyclists. Int $J$ Sports Med 26: 739-746, 2005.

13. Hautier, CA, Linossier, MT, Belli, A, Lacour, JR, and Arsac, LM. Optimal velocity for maximal power production in non-isokinetic cycling is related to muscle fiber type composition. Eur J Appl Physiol 74: 114-118, 1996.

14. Izquierdo, M, Ha"kkinen, K, Gonzalez-Badillo, JJ, Ibanez, $\mathrm{J}$, and Gorostiaga, EM. Effects of long-term training specificity on maximal strength and power of the upper and lower extremities in athletes from different sports. Eur $J$ Appl Physiol 87: 264-271, 2002.

15. Kanehisa, $\mathrm{H}$ and Miyashita, $\mathrm{M}$. Specificity of velocity in strength training. Eur J Appl Physiol 52: 104-106, 1983.

16. Kaneko, M, Fuchimoto, T, Toji, H, and Suei, K. Training effect of different loads on the force-velocity relationship and mechanical power output in human muscle. Scand $J$ Sports Sci 5: 50-55, 1983.

17. Morin, JB, Hintzy, F, Belli, A, and Grappe, F. Forcevelocity relationships and sprint running performances in trained athletes. Sci Sports 17: 78-85, 2002.

18. Murphy, AJ, Wilson, GJ, and Pryor, JF. Use of the isoinertial force mass relationship in the prediction of dynamic human performance. Eur J Appl Physiol 69: 250-257, 1994.

19. Nelson, JR and Thomas, JK. Research Methods in Physical Education. Champaign, IL: Human Kinetics, 1996.

20. Nelson, SG and Duncan, PW. Correction of isokinetic and isometric torque recordings for the effects of gravity. A clinical report. Phys Ther 63: 674-676, 1983.

21. Newton, RU, Kraemer, WJ, Ha"kkinen, K, Humphries, BJ, and Murphy, AJ. Kinematics, kinetics, and muscle activation during explosive upper body movements. J Appl Biomech 12: 31-43, 1996.

22. Perrine, JJ and Edgerton, VR. Muscle force-velocity and power- velocity relationships under isokinetic loading. Med Sci Sports 10: 159-166, 1978.

23. Rahmani, A, Belli, A, Kostka, T, Dalleau, G, Bonnefoy, M, and Lacour, JR. Evaluation of knee

388 Journal of Strength and extensor muscles under non-isokinetic conditions in elderly subjects. J App/Biomech 15: 337-344, 1999.

24. Rahmani, A, Dalleau, G, Viale, F, Hautier, CA, and Lacour, JR. Validity and reliability of a kinematic device for measuring the force developed during squatting. J Appl Biomech 16: 27-36, 2000.

25. Rahmani, A, Viale, F, Dalleau, G, and Lacour, JR. Force/velocity and power/velocity relationships in squat exercise. Eur J Appl Physiol 84: 227-232, 2001.

26. Sargeant, AJ, Hoinville, E, and Young, A. Maximum leg force and power output during short-term dynamic exercise. J Appl Physiol 51: 1175-1182, 1981.

27. Shim, AL, Bailey, ML, and Westings, SH. Development of a field test for upper-body power. J Strength Cond Res 15 192-197, 2001.

28. Taylor, NA, Cotter, JD, Stanley, SN, and Marshall, RN. Functional torque-velocity and power-velocity characteristics of elite athletes. Eur J Appl Physiol 62: 116121, 1991.

29. Terzis, G, Georgiadis, G, Vassiliadou, E, and Manta, P. Relationship between shot put performance and triceps brachii fiber type composition and power production. Eur J Appl Physiol 90: 10-15, 2003.

30. Tihanyi, J, Apor, P, and Fekete, G. Force-velocitypower characteristics and fiber composition in human knee extensor muscles. Eur J Appl Physiol 48: 331343, 1982.

31. van Soest, AJ and Casius, LJ. Which factors determine the optimal pedaling rate in sprint cycling? Med Sci Sports Exerc 32: 1927-1934, 2000.

32. Vandewalle, H, Peres, G, Heller, J, Panel, J, and Monod, H. Force-velocity relationship and maximal power on a cycle ergometer. Correlation with the height of a vertical jump. Eur $J$ Appl Physiol 56: 650-656, 1987.

33. Vincent, WJ. Statistics in Kinesiology. Champaign, IL: Human Kinetics, 1995.

34. Wilson, GJ, Elliott, BC, and Wood, GA. The effect on performance of imposing a delay during a stretch-shorten cycle movement. Med Sci Sports Exerc 23: 364-370, 1991.

35. Wilson, GJ, Murphy, AJ, and Pryor, JF. Musculotendinous stiffness: its relationship to eccentric, isometric, and concentric performance. J Appl Physiol 76: 2714-2719, 1994.

36. Wilson, GJ, Newton, RU, Murphy, AJ, and Humphries, BJ. The optimal training load for the development of dynamic athletic performance. Med Sci Sports Exerc 25: 1279-1286, 1993.

37. Wilson, GJ, Wood, GA, and Elliott, BC. Optimal stiffness of series elastic component in a stretch-shorten cycle activity. $J$ Appl Physiol 70: 825-833, 1991.

38. Winter, DA. Biomechanics and Motor Control of Human Movement. New York: Wiley, 1990.

39. Winter, DA, Wells, RP, and Orr, GW. Errors in the use of isokinetic dynamometers. Eur J Appl Physiol Occup Physiol 46: 397-408, 1981.

40. Young, W, McLean, B, and Ardagna, J. Relationship between strength qualities and sprinting performance. $J$ Sports Med Phys Fitness 35: 13-19, 1995. 